\title{
Quaderni
}

QUADERN I Communication, technologies, pouvoir

81 | Printemps 2013

L'humain médicament

\section{Étude normative comparée : diagnostic préimplantatoire -HLA}

Emmanuelle Rial-Sebbag et Aurélie Mahalatchimy

\section{OpenEdition}

Édition électronique

URL : http://journals.openedition.org/quaderni/716

DOI : 10.4000/quaderni.716

ISSN : 2105-2956

Éditeur

Les éditions de la Maison des sciences de l'Homme

Édition imprimée

Date de publication : 5 juin 2013

Pagination : 77-83

Référence électronique

Emmanuelle Rial-Sebbag et Aurélie Mahalatchimy, «Étude normative comparée : diagnostic préimplantatoire -HLA », Quaderni [En ligne], 81 | Printemps 2013, mis en ligne le 05 mai 2015, consulté le 01 mai 2019. URL : http://journals.openedition.org/quaderni/716 ; DOI : 10.4000/ quaderni.716 


\section{$D$ ossier}

\section{Étude normative Remenciemens}

\section{Genome, Universidad del País Vasco / Euskal}

comparée : diagnostic

Dr. Pilar Nicolas, Chair in Law and the Human Herriko Uniberstitatea.

Dr. Deborah Mascalzoni, EURAC research Center for Biomedicine, Bioethics - ELSI, Bolzano. Dr. Julie Cousineau, Avocate, LL.M., D.C.L, Centre de recherche, Centre hospitalier universitaire Sainte-Justine, Centre de recherche en éthique, Université de Montréal, Centre de recherche en droit public, Université de Montréal. $\mathrm{P}^{\mathrm{r}}$. Roger Browsnword, Professor in Law, founding director of TELOS (Centre for Technology, Ethics and Law in Society), King's College London, United Kingdom.

Human Fertilisation and Embryology Authority, United Kingdom.

\section{Emmanuelle Rial-Sebbag}

Chargée de Recherches UMR U 1027, Inserm, Université Paul Sabatier - Toulouse III

\section{Aurélie Mahalatchimy}

Doctorante en droit, UMR U 1027, Inserm, Université Paul Sabatier - Toulouse III ; IRDEIC, Université

Toulouse 1 - Capitole; Chercheure associée au laboratoire PACTE Département Politique, Organisations UMR 5194
Le diagnostic préimplantatoire associé au typage $\mathrm{HLA}^{1}$ (DPI-HLA) est une technique contemporaine utilisée dans le champ de l'assistance médicale à la procréation. Elle soulève de nombreuses controverses et fait l'objet d'une importante médiatisation. En France, depuis l'adoption de la loi relative à la bioéthique de $2004^{2}$, il est dorénavant possible de proposer aux parents une nouvelle forme de diagnostic préimplantatoire (DPI). Dans les cas où le couple aurait donné naissance à un enfant aîné « atteint d'une maladie génétique entraînant la mort dès les premières années de la vie et reconnue comme incurable au moment du diagnostic », il peut être proposé de faire un DPI sur des embryons obtenus in vitro avant leur réimplantation dans le but d'obtenir des cellules souches hématopoïétiques du sang du cordon qui pourront être utilisées en guise de traitement. Ainsi, «le DPI, associé au typage HLA, a pour objectif de permettre la 
conception d'un enfant indemne de la maladie génétique familiale qui a atteint son aîné(e) et est susceptible de soigner cet aîné(e) malade de façon définitive grâce aux cellules souches $d u$ sang placentaire prélevé dans le cordon ombilical ou, plus tard, de la moelle osseuse ».

En France, cette nouvelle technique, bien peu discutée au Parlement ${ }^{4}$, s'est concrétisée pour la première fois en janvier $2011^{5}$. La naissance de ce bébé a fait l'objet d'une grande couverture médiatique, tout comme celle du premier bébé espagnol en $2008^{6}$. Il est à noter le paradoxe soulevé par ce «battage » médiatique, certes légitime car faisant suite à une avancée scientifique utile pour le traitement de la maladie de l'enfant, et l'absence, du moins en France, d'un large débat sociétal sur l'opportunité d'ouvrir légalement cette voie.

\section{Objectifs}

La possibilité de faire naître un enfant pour soigner son frère ou sa sœur ainé(e), ne relève pas uniquement de la prouesse technique, mais soulève de nombreuses questions éthiques et juridiques fondamentales. A-t-on le droit d'identifier et de « trier » des embryons considérés comme sains (indemnes de la maladie) ? N'est-ce pas une forme moderne d'eugénisme ? Comment évaluer le désir des parents d'avoir un enfant pour lui-même ? Les sources thérapeutiques qui peuvent être utilisées aujourd'hui, sont limitées aux cellules souches du sang de cordon et éventuellement de la moelle osseuse. Pourra-t-on étendre ces sources (ex. aux organes) dans le futur? Quel est le devenir des enfants nés par DPI-HLA dans la fratrie ? Quels sont les gardefous institutionnels qui garantissent la bonne utilisation de cette technique ? Doit-on limiter son utilisation aux seules maladies génétiques?

Pour nous permettre de répondre à ces questions, nous avons souhaité connaître le statut « bioéthique » de cette technique dans plusieurs pays, et non pas limiter cette étude au seul cas français d'ores et déjà largement commenté 7 . Nous avons fait l'hypothèse que l'utilisation de techniques de procréation médicalement assistées en dehors du seul domaine de l'infertilité, visant à éviter la transmission d'une maladie d'une particulière gravité, relevait d'une acceptation sociale dont les limites étaient posées par la loi. De ce fait, nous avons choisi d'investiguer le statut du DPI-HLA dans 4 pays européens ainsi qu'au Québec du fait de son positionnement géographique en Amérique du Nord. Les pays européens ont, quant à eux, été choisis en fonction de leurs approches culturelles différentes au regard de la bioéthique ainsi que de notre réseau de correspondants dans ces pays : France, Royaume-Uni, Espagne et Italie.

\section{Méthodologie}

Afin d'analyser le statut « bioéthique » du DPIHLA dans les pays choisis, nous avons élaboré un court questionnaire qui nous a permis, à travers 5 questions, de connaître 1) le régime juridique de la technique, 2) l'état de l'art en pratique, 3) les débats sociétaux actuels et les questions non résolues. Ces questionnaires ont été remplis par des juristes/éthiciens spécialistes du domaine dans chacun des pays concernés.

\section{Résultats et discussion}

Les réponses peuvent être matérialisées comme 
ci-dessous :

\begin{tabular}{|l|l|l|l|l|l|}
\hline & France & Royaume-Uni & Italie & Espagne & Québec \\
\hline CADRE JURIDIQUE & $\begin{array}{l}\text { OUI } \\
2004\end{array}$ & $\begin{array}{l}\text { OUI } \\
2008\end{array}$ & $\begin{array}{l}\text { OUI (implicite) } \\
2004\end{array}$ & $\begin{array}{l}\text { OUI } \\
2006\end{array}$ & $\begin{array}{l}\text { OUI (implicite) } \\
2010\end{array}$ \\
\hline REGIME & $\begin{array}{l}\text { Autorisation } \\
\text { sous condi- } \\
\text { tions }\end{array}$ & $\begin{array}{l}\text { Interdiction } \\
\text { avec déroga- } \\
\text { tion }\end{array}$ & Interdiction & $\begin{array}{l}\text { Autorisation } \\
\text { sous condi- } \\
\text { tions }\end{array}$ & $\begin{array}{l}\text { Interdiction } \\
\text { implicite }\end{array}$ \\
\hline PRATIQUE & OUI & OUI & $\begin{array}{l}\text { NON mais cas } \\
\text { rapportés de } \\
\text { conception } \\
\text { ailleurs }\end{array}$ & OUI & NON \\
\hline $\begin{array}{l}\text { EVOLUTION DU } \\
\text { REGIME }\end{array}$ & NON & NON & $\begin{array}{l}\text { OUI sur la loi } \\
\text { relative à la } \\
\text { fertilité }\end{array}$ & NON & $\begin{array}{l}\text { NON loi } \\
\text { récente }\end{array}$ \\
\hline AVIS DE COMITE \\
$\begin{array}{l}\text { D'ETHIQUE } \\
\text { Oui avis }\end{array}$
\end{tabular}

Une première analyse générale de ces résultats a permis de mettre en évidence l'état du débat bioéthique. La question fondamentale de l'autorisation ou de l'interdiction du DPI-HLA ne dépend pas de la disponibilité de la technique. Cette dernière existe, est validée, et peut être potentiellement mise en œuvre dans l'ensemble des pays choisis pour l'étude. Cependant, deux pays l'interdisent (Italie et Canada (Québec)) et ce uniquement par la mise en avant d'arguments moraux. Le choix de l'inscription juridique sous un régime (autorisation sous conditions, interdiction assortie de dérogations) ou l'autre (interdiction totale) répond à un difficile arbitrage entre différents principes. Que doit-on privilégier socialement entre l'intérêt thérapeutique d'un enfant malade (le receveur) et la nature sacrée de l'enfant à naître, envisagé comme sourcethérapeutique ? Comment justifier de faire perdre une chance de guérison à l'enfant-receveur alors même que la technique est disponible et que cette dernière est peu invasive (du moins pour les cellules issues du sang de cordon) ? Comment assurer la qualité de vie future de l'enfant-source au sein de la fratrie?

Finalement, le choix de l'une ou l'autre des postures s'est essentiellement réalisé sur deux séries d'arguments. Dans les pays qui autorisent le DPI-HLA, le débat bioéthique a été balayé et 
la technique justifiée par la qualité de sa mise en œuvre et la sécurité des procédures (1). A contrario, ce sont essentiellement sur des arguments bioéthiques que se sont fondés les régimes d'interdiction (2).

\section{La stabilisation technique de la pratique: des outils au service de la santé publique}

Dans les trois pays où il est possible de pratiquer le DPI-HLA, la loi réserve son utilisation à un champ d'application strict ce qui atteste l'existence de dérive potentielle et la nécessité de s'en prémunir. La France exige ainsi que le DPI-HLA soit uniquement utilisé pour éviter la transmission «d'une maladie génétique entraînant la mort dès les premières années de la vie et reconnue comme incurable au moment du diagnostic $\rangle^{8}$. Il en va de même pour l'Espagne qui autorise le diagnostic exclusivement pour la «détection d'une maladie héréditaire grave, d'apparition précoce et susceptible d'un traitement curatif post-natal $»^{9}$. Le Royaume-Uni est plus vague tant pour la mise en œuvre du diagnostic qui peut se faire dès «qu'une condition médicale sérieuse » est avancée, que pour les sources thérapeutiques utilisées dans le futur ${ }^{10}$. En effet, alors que la loi française spécifie bien que seules pourront être utilisées les cellules souches du sang de cordon et éventuellement celles de la moelle osseuse ${ }^{11}$, le Royaume-Uni étend les sources possibles à « d'autres» tissus en précisant seulement qu'il ne peut s'agir d'un organe dans son entier ${ }^{12}$.

De manière plus opérationnelle, les législations autorisant le DPI-HLA combinent des mesures permettant de s'assurer de la qualité des établissements et de la traçabilité de leurs activités (a) et des mesures d'évaluation de la demande d'accès à la technique (b) :

a) Dans tous les pays qui autorisent le DPI-HLA ( $\mathrm{Fr}, \mathrm{Ru}, \mathrm{Es}$ ), les établissements doivent avoir une autorisation pour mettre en œuvre cette technique. Cette autorisation a pour objectif de démontrer le savoir-faire des équipes et notamment leur caractère pluridisciplinaire. Les instances chargées de délivrer l'autorisation d'exercice de ces établissements doivent également s'assurer de la bonne application des règles juridiques au sein des services. Ce dernier point est particulièrement important quand on sait que l'obligation de démontrer le caractère grave ou sérieux de la maladie pour laquelle on souhaite mettre en œuvre un DPI-HLA repose sur les équipes de soins. Cette responsabilité des soignants est d'autant plus grande quand le législateur n'a pas souhaité mettre en place de liste de maladies génétiques (cas de la France).

b) Le principe du recours au DPI-HLA est acquis. Ces pratiques sont formalisées par écrit et doivent faire l'objet d'une évaluation dans les trois pays (Fr, Ru, Es). Ce sont des Agences nationales qui sont en charge de mener cette évaluation ${ }^{13}$ au cas par cas selon une procédure systématique. Le Royaume-Uni déroge légèrement à cette approche systématique en distinguant deux cas de figures :

- soit le cas à traiter a déjà un précédent (liste consultable ${ }^{14}$ sur le site l'Agence compétente : la Human Fertilisation and Embryology Authority (HFEA)) et une approbation simple et rapide par un panel de la HFEA composé de personnel exécutif suffit ; 
- soit le cas à traiter est vu pour la première fois au Royaume-Uni, il est alors considéré comme pouvant soulever de nouvelles controverses et sera évalué par un comité d'autorisation de l'agence. Dans tous les cas le clinicien qui traite l'enfant doit soutenir le projet de DPI-HLA et doit motiver ce projet.

À ces éléments de procédure, doit être également ajouté le débat sociétal afin d'évaluer le poids de ce dernier sur la règlementation adoptée dans les pays étudiés.

\section{La stabilisation éthique des pratiques : du débat sociétal à la réglementation}

Au-delà de l'autorisation ou de l'interdiction de la pratique dans les pays étudiés, nous avons souhaité étendre notre étude aux liens qu'avaient entretenus, et qu'entretenaient encore, les règles juridiques et les débats de société autour de la pratique du DPI-HLA. Deux approches ont émergé de notre analyse, distinguant, dans ce cas aussi, les pays dans lesquels le DPI-HLA était autorisé (a) et ceux dans lesquels il était interdit (b) :

a) La France et le Royaume-Uni ${ }^{15}$ ont certes encadré juridiquement la pratique du DPIHLA dans le sens de son autorisation, mais les trajectoires de cette inscription juridique ont été bien différentes. La France n'a adopté cette possibilité que de manière incidente après un débat parlementaire à la marge. Les arguments soulevés durant l'unique session de discussion au Parlement étaient essentiellement relatifs à l'instrumentalisation de l'enfant. À ce titre, les débats ont notamment dû répondre à la question suivante : «comment envisager qu'un enfant puisse être considéré non plus comme une fin, mais comme un moyen ? ». Le législateur, tout en se basant sur le droit de l'enfant à naître, a ainsi choisi d'arbitrer en faveur du principe thérapeutique et a légalisé la pratique sous couvert du respect de conditions strictes ${ }^{16}$. Au RoyaumeUni, c'est à la suite d'une question de compétence de la HFEA que la question de l'autorisation de cette technique a été tranchée. En 2001, la HFEA avait autorisé des parents d'enfants gravement malades à recourir à un DPI-HLA pour avoir un autre enfant. Ces autorisations ont été remises en cause dans l'affaire Quintavalle ${ }^{17}$ au regard de la compétence de la HFEA, ce qui de facto remettait en cause la légalité de la technique. En 2005, la Chambre des Lords a décidé que cette autorisation relevait bien de la compétence de la HFEA et par là même a rendu la pratique licite sous respect de conditions posées ultérieurement par la loi en 2008.

b) Les arguments bioéthiques ont été fondateurs des décisions prises dans les deux autres pays étudiés d'interdire le recours au DPI-HLA. En premier lieu, l'Italie doit faire face à un débat vigoureux sur le recours au DPI en général. Cette technique n'est autorisée pour aucune indication ; elle est donc interdite a fortiori pour le DPIHLA. Avançant la nature sacrée de la vie et de l'embryon, ainsi que les risques d'eugénisme liés à l'autorisation d'une telle pratique, le législateur italien a consacré le principe d'interdiction. Ce principe doit faire face à une remise en cause juridique par la Cour Européenne des droits de l'homme. En effet par un arrêt du 28 août 2012, la Cour a condamné l'Italie pour sa législation qui interdit le DPI sur un embryon. L'argument principal de cet arrêt repose sur l'incohérence 
de la législation italienne entre le DPI - interdit - et l'avortement thérapeutique - autorisé ${ }^{18}$. Au Québec, le débat s'est orienté différemment, notamment du fait que le DPI y est autorisé. En revanche, les indications pour lesquelles un tel diagnostic peut être posé sont strictement limitées à l'établissement d'une analyse visant à identifier une maladie monogénique grave ou une anomalie chromosomique ${ }^{19}$. Un DPI-HLA ne peut pas s'entendre de la sorte puisqu'il se résume à un test de compatibilité HLA, ce qui emporte de facto l'interdiction de cette pratique au Québec. Toutefois les enjeux éthiques soulevés par le DPIHLA ont été discutés notamment en balançant l'intérêt thérapeutique pour l'enfant à soigner versus l'instrumentalisation de l'enfant à naître. Ces éléments de discussion sont très présents tant dans le rapport de la Commission de l'éthique en science et en technologie du Québec rendu en $2009^{20}$ que dans la synthèse de la consultation publique $^{21}$ réalisée pour ce même rapport. Les arguments présentés convergent ainsi en faveur du principe d'interdiction.

En conclusion, les postures entre les pays étudiés sont inconciliables concernant la légalisation ou l'interdiction du DPI-HLA. Balançant entre respect de la vie humaine et chance d'être traité, la technique n'est pas uniformément disponible dans les pays européens notamment en raison d'obstacles épistémologiques. On peut cependant relever une notion sur laquelle l'ensemble des législations tend à s'accorder : il faut systématiquement s'assurer que la technique ne soit pas associée à l'instrumentalisation de l'enfant à naître. Bien évidemment, cet impératif reste bien plus simple à respecter dans les cas où l'interdiction est formelle que dans les cas où l'enfant est déjà né, puisque cela implique d'élaborer des mesures de surveillance et de s'assurer de cette non-instrumentalisation dans le futur de l'enfant. 
$\mathrm{N} \cdot \mathrm{O} \cdot \mathrm{T} \cdot \mathrm{E} \cdot \mathrm{S}$

1. Communément appelé «bébé-médicament»ou encore « bébé du double espoir ».

2. Art. L2131-4-1, du code de la santé publique (CSP), Loi relative à la bioéthique de 2004 .

3. Définition donnée par l'Agence de la Biomédecine, http://www.agence-biomedecine.fr/Extension-du-DPIDPI-HLA, 179 .

4. L'insertion du DPI-HLA dans le projet de Loi de bioéthique s'est faite au moyen de l'adoption d'un amendement, après discussion, au cours de la $2^{\mathrm{e}}$ séance de discussion à l'Assemblée nationale le 10 décembre 2003, http://www.assemblee-nationale.fr/12/cri/20032004/20040096.asp\#PG24

5. http://www.lemonde.fr/societe/article/2011/02/07/ le-premier-bebe-medicament-francais-estne_1476586_3224.html.

6. http://www.europapress.es/salud/salud-bienestar/ noticia-primer-bebe-medicamento-logra-salvarhermano-20090313165645.html .

7. Voir notamment Aline Cheynet de Beaupré Terrasson de Fougères, "Le bébé du double espoir », Recueil Dalloz Sirey, 03/03/2011, n 9, pp. 603-604 ; Jean-René Binet, « Le double DPI pérennisé par l'Assemblée nationale », JCP G Semaine Juridique (édition générale), 21/02/2011, nº, p. 370.

8. Loi de bioéthique 2011.

9. Ley 14/2006 14/2006, de 26 de mayo, sobre técnicas de reproducción humana asistida (BOE, núm.126 Sábado 27 mayo 2006)).

10. Schedule 2, 1ZA (1) (d) Human Fertilisation and Embryology Act 2008.

11. Ceci résulte des dispositions relatives au don de cellules quand les donneurs sont des mineurs vivants, articles L 1241-1, 2 et 3 du CSP.

12. HFEA act 2008 Schedule 2 Embryo testing 1ZA4),
In sub-paragraph (1) (d) la référence à d'autres tissus provenant de l'enfant conçu n'inclut pas l'accès à un organe dans son entier.

13. Agence de la Biomédecine en France, agences sanitaires compétentes après avis de la Commission nationale de l'aide médicale à la procréation en Espagne et l'autorité pour la fertilité humaine et l'embryologie en Angleterre.

14. HFEA's website, http://www.hfea.gov.uk/cps/hfea/ gen/pgd-screening.htm.

15. Nous n'avons pas recueilli de données pour l'Espagne.

16. Article L2131-4-1 du CSP.

17. House of Lords, Quintavalle v. Human Fertilisation and Embryology Authority, [2005] UKHL 28. Voir notamment : R. Brownsword, Chapter 6 «The Challenge of Regulatory Connection » in Rights, Regulation, and the Technological Revolution, OUP Oxford, 6 mars 2008.

18. CEDH, 28 août 2012, Costa et Pavan c. Italie, $\mathrm{n}^{\circ} 54270 / 10$.

19. Loi sur les activités cliniques et de recherche en matière de procréation assistée, L.R.Q., chapitre A-5.01 2009 et règlement sur les activités cliniques en matière de procréation assistée, R.R.Q., c. A-5.01, r. 1, 2010. 20. Éthique et procréation assistée : des orientations pour le don de gamètes et d'embryons, la gestation pour autrui et le diagnostic préimplantatoire, Québec, 2009.

21. Commission de l'éthique en science et en technologie, Consultation publique électronique sur la procréation assistée : Rapport adressé à la Commission de l'éthique de la science et de la technologieSynthèse d'une étude réalisée par Florence Piron et Julie Dussault, Département d'information et de communication, Groupe de recherche-action sur la participation et la consultation publique, Université Laval, Québec, 2009. 
